\title{
Organic grafts in sacral colpopexy; are they introduced prematurely? Long term results for 29 patients
}

Received: 16 July 2005 / Accepted: 9 August 2005 / Published online: 9 September 2005

(C) Springer-Verlag Berlin / Heidelberg 2005

\begin{abstract}
Sacral colpopexy with non-resorbable grafts is successful in $85-100 \%$ of cases. The main drawback of these materials is the substantial risk of erosion of the mesh in $0.6-8.8 \%$ of cases. With allogenic materials, for example fascia lata, dura mater, and (bovine) pericard, there is no risk of erosion. Studies in dogs indicate that the material is incorporated within 3-6 months, while inducing collagen formation - the graft is actually substituted by the recipient's own collagen. In this study solvent-dried fascia lata, dura mater, and bovine pericard were used as implants for sacral colpopexy in patients with vaginal vault prolapse. Long-term anatomical results are reported. In a retrospective observational study 29 patients with vaginal vault prolapse underwent sacral colpopexy. Patient characteristics were recorded on standardized clinical record forms. We used Tutoplast homologous fascia lata and dura mater or bovine pericard. In September 2001 all patients who had not had a second colpopexy in the meantime were invited to visit our outpatient clinic for gynecological examination. Surgical failure was defined as a second colpopexy or a vaginal vault prolapse or enterocele up to or beyond the hymen. After a mean follow up period of 38.2 months, failure based on the anatomical result occurred in 11 of 29 patients (38\%); success was doubtful in another four cases $(14 \%)$. For fourteen patients $(48 \%)$ the sacral colpopexy was a success. Low parity and former prolapse surgery may be indicators of likely failure of the graft in sacral colpopexy. Neither surgical approach (laparotomy
\end{abstract}

M. H. Kerkhof · H. A. M. Brölmann (凶)

VU University Medical Center, De Boelelaan 1117,

1181HV Amsterdam, The Netherlands

E-mail: HansBrolmann@vumc.nl

Tel.: + 31-20-4444851

Fax: $+31-20-4443333$

P. J. A. G. Geomini

Saint Joseph Hospital, PO Box 7777,

$5500 \mathrm{MB}$ Veldhoven,

The Netherlands or laparoscopy) nor other surgical factors affected results significantly. Organic materials, for example fascia lata, dura mater, and bovine pericard, as alternatives are not suitable for sacral colpopexy because of a substantial risk of graft failure.

Keywords Sacral colpopexy - Vaginal vault prolapse · Laparoscopy $\cdot$ Organic graft $\cdot$ Sacral

\section{Introduction}

In 1926, for the first time in a human being, organic allografts were used in hernia repair [1]. In gynecological surgery allografts are mainly used in sacral colpopexy, a procedure intended to repair vaginal vault prolapse (after hysterectomy) or uterine prolapse. The vaginal apex is attached to the promontory or to the hollow of the sacrum. If non-resorbable grafts, for example GoreTex, Prolene, Mersylene or Marlex, are used the repair is successful in $85-100 \%$ of cases. [2-5]. The main drawback of these materials is the substantial risk of erosion of the mesh. This serious complication occurs in 0.6 $8.8 \%$ of cases [6] and usually requires removal of the graft, vaginally or abdominally. For many years allogenic organic materials, for example fascia lata, have been used for reconstructive purposes in the human body, mainly in orthopaedic surgery. In cases of anterior cruciate ligament defects [7], in suspensions of the shoulder, ankle, or hip, and the repair of tendons or ligaments [8] fascia lata has been used successfully. Studies in dogs indicate that this material is incorporated within 3-6 months, while inducing collagen formation [9].

The graft is actually replaced by the recipient's own collagen. One of the advantages is the absence of any risk of expulsion of the implanted material. No studies of humans have been performed to evaluate the longterm effectiveness of organic allografts in sacral colpopexy, however. 
In this study, solvent-dried fascia lata, dura mater, and bovine pericard were used as implants for sacral colpopexy in patients with uterine or vaginal vault prolapse. Long-term anatomical results are reported.

\section{Materials and methods}

From January 1996 until May 2000 we performed sacral colpopexy, using organic, resorbable grafts, on 29 patients with vaginal vault prolapse. The surgery was performed at the Department of Obstetrics and Gynaecology in Saint Joseph Hospital, Veldhoven, The Netherlands, a community-based teaching hospital with 500 beds. Patients with failed sacral colpopexy in their medical history were excluded from the study. All patients were asked for consent to be in the surgical database.

Patient characteristics, i.e. age, parity, body mass index (BMI), former gynecological surgery, and data about the surgical technique, the graft material used, additional procedures, operating time, and perioperative and postoperative complications were collected from the hospital record files, and recorded on standardized clinical record forms.

Surgical technique: until June 1998 all procedures were performed using a Pfannenstiel incision. In the second half of the study all cases were performed by laparoscopy. In laparotomy the bowel was packed off, the vagina was elevated with a pelotte, and the peritoneal surface was resected from the vaginal apex. The laparoscopy was performed through four ports (two $5 \mathrm{~mm}$, two $10 \mathrm{~mm}$ ). In both approaches the graft was sutured to the posterior, sometimes to the anterior, site of the vault, using polydioxanone sutures (PDS) in laparotomy and Ethibond sutures (Johnson\&Johnson, Ethicon, Cincinnati, USA) in laparoscopy. In laparotomy the graft was attached to the promontory by polydioxanone sutures, in laparoscopy by hernia staples (Johnson\&Johnson, Endosurgery, Cincinnati, USA) without undue tension. In cases of urodynamically confirmed stress incontinence, the sacral colpopexy was combined with a colposuspension according to Burch, by laparotomy or by a laparoscopic approach. If a rectocele was present, a posterior colporrhaphy was performed. Peritonisation of the graft was not performed, and the pouch of Douglas was not closed on a routine basis. All procedures were performed by one of the authors (HB).

We used Tutoplast homologous fascia lata and dura mater or bovine pericard. (Tutogen Medical, Neunkirchen am Brand, Germany). The homologous graft material was collected within $24 \mathrm{~h}$ of the death of a human donor and preserved according to a treatment protocol to inactivate bacteria and other pathogens, for example conventional and unconventional viruses and prions. Solvent-dehydrated material sterilized using gamma radiation is thought to be a more appropriate grafting material than freeze-dried specimens obtained from tissue banks [10].

In September 2001 all patients who had not had a second colpopexy in the meantime were invited to visit our outpatient clinic for gynecological examination and to complete a questionnaire. Surgical failure was defined as a second colpopexy or a vaginal vault prolapse or enterocele up to or beyond the hymen. In cases of relaxation of another site of the vagina up to or beyond the hymen that could be redressed by stretching the vagina, the success was considered doubtful.

One clinical investigator examined the patients. The extent of prolapse was scored according to the Halfway Grading System as described by Baden and Walker [11] and was compared with the score according to the standard system of terminology of the International Continence Society [12]. Statistical analysis (Chi squared and Fisher exact) was performed using SPSS 9.0 statistical software. $P<0.05$ was considered to be significant.

\section{Results}

During a mean follow up period of 38.2 months (SD 16.7), failure occurred in 11 of 29 patients $(38 \%)$, success

Table 1 Patient characteristics (age, parity and body mass index, BMI) and results from sacral colpopexy with organic graft for 29 patients

\begin{tabular}{lllll}
\hline & Success $(n=14)$ & Failure $(n=11)$ & Doubtful $(n=4)$ & Total $(n=29)$ \\
\hline Age (SD) & $60.2(13)$ & $60.2(11)$ & $49.1(5)$ & $58.7(10.9)$ \\
Parity (SD)* & $3.2(1.0)$ & $2.6(1.7)$ & $1.7(1.0)$ & $22.4(3.2)$ \\
Body mass index & $25.8(3.3)$ & $25.9(2.3)$ & & 2.8 \\
Former prolapse surgery* & & & 3 & 11 \\
Yes & 2 & 6 & 1 & 18 \\
No & 12 & 5 & & \\
Menopausal state & 3 & & 3 & \\
Pre & 11 & 2 & 1 & 21 \\
Post & & 9 & & \\
\hline
\end{tabular}

$* P<0.05$ 
was doubtful in another four cases $(14 \%)$. On the basis of the anatomical result the sacral colpopexy was a success for fourteen patients $(48 \%)$ ). The characteristics of the patients according to the postoperative results are shown in Table 1. Age, BMI, and menopausal status had no impact on results. For patients with parity $>2$, however, the sacral colpopexy was successful in 12 of 17 cases $(71 \%)$ whereas for patients with parity 2 or less the operation was a success for only 2 of $12(17 \% ; P=0.01)$. Also, former prolapse surgery impaired results significantly. If patients had undergone vaginal prolapse surgery (anterior or posterior colporrhaphy) in the past, the sacral colpopexy succeeded in 2 of 11 cases $(18 \%)$. Without this medical history success was achieved in 12 of $18(67 \%)$ patients $(P=0.03)$.

Operating time, blood loss, combined surgery, complications, and the graft material did not affect results significantly (Table 2), and no difference was found between patients who had a laparoscopy or a laparotomy as surgical approach. Surgical obliteration of the Douglas pouch may have some effect on results. After closure of the pouch success was achieved in 5 of 8 cases $(62.5 \%)$, without closure in 9 of $21(43 \%)$ $(P=0.10)$.

As points $\mathrm{D}$ and $\mathrm{C}$ in the ICS score [12] are the points representing the apex of the vagina, the score in the absence of a vault prolapse is equal to the length of the vagina minus $2 \mathrm{~cm}$, which is the mean distance between the hymen and the vaginal outlet. So the formula (total vaginal length-2+D or C) is zero in the absence of prolapse but can reach the length of the vagina in cases of a grade 4 prolapse of the vaginal vault (total eversion). If the cut off value is modified from 1 to 3 a maximum kappa value of 0.39 is achieved in cases of a cut off value of 2 . This kappa value increases up to 0.43 if the doubtful cases are considered a success. If clinical assessment of the vaginal vault according to Baden and Walker is used as a reference test, the area under the ROC curve (not shown) for point $\mathrm{D}$ is 0.78 (CL $0.59-0.97$ ) and for point $\mathrm{C}$ is 0.76 (CL $0.56-0.95)$.

\section{Discussion}

In this retrospective observational study of 29 patients who underwent a sacral colpopexy with organic, resorbable graft, anatomical outcome was shown to be successful for 14 patients $(48 \%)$ only after a mean follow-up period of 38 months. We deliberately chose anatomical outcome rather than functional complaints, because they are considered multifactorial. If the vaginal vault is not fixed during Valsalva maneuver, detachment of the graft is likely.

In our hands the prolapse score according to the ICS did not correlate well with the clinical judgment with the "half-way grading system" of Baden and Walker. Although we realize that two different investigators, blind to the results of the other, would have strengthened the results, we believe it is impossible not to score the half-way grading system while scoring according to the ICS. We therefore decided to present the results anyway. Difficulty in finding the hymenal ring and distinguishing points $\mathrm{C}$ and $\mathrm{D}$ in hysterectomized patients perhaps make the ICS score less suitable for assessing vaginal vault prolapse.

If anatomical endpoints are assessed, long term effectiveness of $90 \%$ or more is usually reported [3]. Timmons et al. [2] performed a abdominal sacral colpopexy on 163 women for correction of vaginal vault prolapse from 1972 to 1989. The patients were followed for 9 months to 18 years, with a median follow-up of 33 months. They reported good vaginal vault support, assessed by gynecological examination, in as many as $161(99 \%)$ of the patients. In a more recent study Webb et al. [5] reported, after a median follow up of 15.8 years (range 0.4-48.4), a failure rate, assessed by recurrent functional complaints (bulge, protrusion), of 80 out of

Table 2 Operation characteristics and results from sacral colpopexy with organic graft for 29 patients

\begin{tabular}{|c|c|c|c|c|c|}
\hline & & Success $(n=14)$ & Failure $(n=11)$ & Doubtful $(n=4)$ & Total $(n=29)$ \\
\hline \multicolumn{2}{|c|}{ Operating time in $\min (\mathrm{SD})$} & $145(30)$ & $144(53)$ & $135(33)$ & $143(39)$ \\
\hline \multicolumn{2}{|c|}{ Blood loss in $\mathrm{mL}$ (SD) } & $271(14)$ & $181(11)$ & $262(4)$ & $236(29)$ \\
\hline \multirow[t]{2}{*}{ Combination surgery } & Yes & 12 & 5 & 2 & 19 \\
\hline & No & 2 & 6 & 2 & 10 \\
\hline \multirow[t]{2}{*}{ Complications during } & Yes & 1 & 3 & 0 & 4 \\
\hline & No & 13 & 8 & 4 & 25 \\
\hline \multicolumn{6}{|l|}{ Surgical approach } \\
\hline \multicolumn{2}{|l|}{ Laparotomy } & 6 & 5 & 2 & 13 \\
\hline \multirow{2}{*}{\multicolumn{6}{|c|}{$\begin{array}{l}\text { Laparoscopy } \\
\text { Material }\end{array}$}} \\
\hline & & & & & \\
\hline \multicolumn{2}{|l|}{ Dura mater } & 4 & 2 & 3 & 9 \\
\hline \multicolumn{2}{|l|}{ Fascia lata } & 4 & 7 & 1 & 12 \\
\hline \multicolumn{2}{|l|}{ Bovine pericard } & 6 & 2 & 0 & 8 \\
\hline \multicolumn{6}{|c|}{ Enterocele prophylaxis (Halban, McCall) } \\
\hline \multicolumn{2}{|c|}{ Yes } & 5 & 2 & 1 & 8 \\
\hline \multicolumn{2}{|l|}{ No } & 9 & 9 & 3 & 21 \\
\hline
\end{tabular}


$504(16 \%)$, but only $5 \%$ had a subsequent prolapse repair. Benson et al. [4] defined "optimal surgical effectiveness" if the woman was asymptomatic, the apex did not descend more than $50 \%$ of its length, or the vaginal wall did not protrude beyond the hymen. With this definition, after a mean follow up period of 30 months, they reported surgical effectiveness of $58 \%$. This low effectiveness may be explained by the combination of subjective and objective criteria.

We decided to use organic resorbable material for the sacral colpopexy after being made aware of publications on its use $[13,14]$. In these publications follow up of results was not described [13], neither were the results correlated with the different materials [14]. Erosion of the graft was never reported, however, and replacement of the graft with collagen was generally accepted from animal studies and orthopaedic use. Our initial experience with the material in 12 patients has been published elsewhere [15]. The results did not differ significantly from results for 28 patients for whom Goretex mesh was used in the operation, although the numbers were small. In a later publication the failure of allograft suburethral slings was reported in 7 of 35 cases $(20 \%)$ [16]. Histology of the graft, retrieved at re-operation, showed degeneration in two patients or total absence in five. From that time we have used non-resorbable material for sacral colpopexies.

One can speculate on the reason for organic graft failure in sacral colpopexy. The mechanical properties depend on the metabolic phase of the graft material-degeneration, revascularization, fibroplasia, and maturation of the collagen. During maturization vascularity and cellularity decrease and nuclei are arranged longitudinally. In this way the allograft serves as a scaffold for collagen formation, while it is fully substituted by vital collagen. Freeze-dried fascia lata in the reconstruction of the anterior cruciate ligament in dogs is complete in a mean time of 29 months [7]. During this process the breaking load in biomechanical testing changes [9]. In a experiment with dogs the breaking load of the anterior cruciate ligament 3 weeks after implantation was equal to that of the contralateral ligament. After 6 weeks the breaking load of the operated side was only $19 \%$ compared with the contralateral side, after 12 weeks $14 \%$ and after 24 weeks $51 \%$. Because it is impossible to prevent full stress on the implanted graft during recovery after sacral colpopexy (as a consequence of coughing, vomiting, constipation, walking, etc.), the graft - in the phase of reduced strength - may not withstand these forces and may break down.

In the current study the sacral colpopexy failed more frequently in patients who had previous vaginal prolapse surgery $(67 \%$ versus $18 \%)$ or had lower parity. If we attribute a certain predisposition, caused by weak collagen formation, to these patients, maybe collagen induction by the organic graft is insufficient, resulting in little strength of the final tissue. Other explanations are also possible - degradation of the graft by the vaginal bacterial flora, the relatively rich blood supply in the vagina, or host-vs-graft reactions [16]. Only formal animal studies can reveal the behavior of organic grafts used under stress in the peritoneal cavity.

\section{References}

1. Koontz AR (1926) Experimental results in the use of dead fascia grafts for hernia repair. Ann Surg 83:523-536

2. Timmons MC, Addison WA, Addison SB, Cavenar MG (1992) Abdominal sacral colpopexy in 163 women with posthysterectomy vaginal vault prolapse and enterocele. Evolution of operative techniques. J Reprod Med 37:323-327

3. Toozs-Hobson P, Boos K, Cardozo L (1998) Management of vaginal vault prolapse. Br J Obstet Gynaecol 105:13-17

4. Benson JT, Lucente V, McClellan E (1996) Vaginal versus abdominal reconstructive surgery for the treatment of pelvic support defects: a prospective randomized study with longterm outcome evaluation. Am J Obstet Gynecol 175:14181421

5. Webb MJ, Aronson MP, Ferguson LK, Lee RA (1998) Posthysterectomy vaginal vault prolapse: primary repair in 693 patients. Obstet Gynecol 92:281-285

6. Visco AG, Weidner AC, Barber MD, Myers ER, Cundiff GW, Bump RC, Addison WA (2001) Vaginal mesh erosion after abdominal sacral colpopexy. Am J Obstet Gynecol 184:297302

7. Horstman JK, Ahmadu-Suka F, Norrdin RW (1993) Anterior cruciate ligament fascia lata allograft reconstruction: progressive histologic changes toward maturity. Arthroscopy 9:509518

8. Bright RW, Green WT (1981) Freeze-dried fascia lata allografts: a review of 47 cases. J Pediatr Orthop 1:13-22

9. Curtis RJ, Delee JC, Drez DJ Jr (1985) Reconstruction of the anterior cruciate ligament with freeze dried fascia lata allografts in dogs. A preliminary report. Am J Sports Med 13:408414

10. Hinton R, Jinnah RH, Johnson C, Warden K, Clarke HJ (1992) A biomechanical analysis of solvent-dehydrated and freeze-dried human fascia lata allografts. A preliminary report. Am J Sports Med 20:607-612

11. Baden WF, Walker T (1992) Fundamentals symptoms and classification. In: Baden WF, Walker T (eds) Surgical repair of vaginal defects J.B. Lippincott Company, Philadelphia, pp 9 23

12. Bump RC, Mattiasson A, Bo K et al (1996) The standardization of terminology of female pelvic organ prolapse and pelvic floor dysfunction. Am J Obstet Gynecol 175:10-17

13. Lansman HH (1984) Posthysterectomy vault prolapse: sacral colpopexy with dura mater graft. Obstet Gynecol 63:577-82

14. Virtanen H, Hirvonen T, Makinen J, Kiilholma P (1994) Outcome of thirty patients who underwent repair of posthysterectomy prolapse of the vaginal vault with abdominal sacral colpopexy. J Am Coll Surg 178:283-287

15. Geomini PJAG, Brölmann HAM, van Binsbergen HJM, Mol BW (2001) Vaginal vault suspension by abdominal sacral colpopexy for prolapse: a follow up study of 40 patients. Eur J Obstet Gynaecol Reprod Biol 94:234-238

16. Fitzgerald MP, Mollenhauer J, Bitterman P, Brubaker L (1999) Functional failure of fascia lata allografts. Am J Obstet Gynecol 181:1339-44

17. Lecuru F, Taurelle R, Clouard C, Attal JP (1994) Surgical treatment of genito-urinary prolapses by abdominal approach. Results in a continuous series of 203 operations. Ann Chir 48:1013-1019

18. Marchionni M, Bracco GL, Checcucci V et al (1999) True incidence of vaginal vault prolapse. Thirteen years of experience. J Reprod Med 44:679-684

19. Barrington JW, Edwards G (2000) Posthysterectomy vault prolapse [In Process Citation]. Int Urogynecol J Pelvic Floor Dysfunct 11(4):241-245 